ARTICLE OPEN

\title{
Inhibition of Axin1 in osteoblast precursor cells leads to defects in postnatal bone growth through suppressing osteoclast formation
}

\author{
Bing Shu ${ }^{1,2,3}$, Yongjian Zhao ${ }^{1,2,3}$, Shitian Zhao ${ }^{1,2,3}$, Haobo Pan ${ }^{4}$, Rong Xie ${ }^{5}$, Dan Yi ${ }^{4}$, Ke Lu (iD ${ }^{5}$, Junjie Yang ${ }^{1,2,3}$, Chunchun Xue ${ }^{1,2,3}$, \\ Jian Huang $\mathbb{D}^{5}$, Jing Wang ${ }^{1,3}$, Dongfeng Zhao ${ }^{1,2,3}$, Guozhi Xiao ${ }^{6}$, Yongjun Wang ${ }^{1,2,3}$ and Di Chen ${ }^{4}$
}

\begin{abstract}
Axin1 is a negative regulator of $\beta$-catenin signaling and its role in osteoblast precursor cells remains undefined. In the present studies, we determined changes in postnatal bone growth by deletion of Axin 1 in osteoblast precursor cells and analyzed bone growth in newborn and postnatal Axin $1^{\text {Osx }}$ mice and found that hypertrophic cartilage area was largely expanded in Axin $1^{\text {Osx }} \mathrm{KO}$ mice. A larger number of chondrocytes and unabsorbed cartilage matrix were found in the bone marrow cavity of $A x i n 1^{\text {Osx }} \mathrm{KO}$ mice. Osteoclast formation in metaphyseal and subchondral bone areas was significantly decreased, demonstrated by decreased TRAPpositive cell numbers, associated with reduction of MMP9- and cathepsin K-positive cell numbers in Axin ${ }^{\text {Osx }}$ KO mice. OPG expression and the ratio of Opg to Rankl were significantly increased in osteoblasts of Axin $1^{\text {Osx }} \mathrm{KO}$ mice. Osteoclast formation in primary bone marrow derived microphage (BMM) cells was significantly decreased when BMM cells were cultured with conditioned media (CM) collected from osteoblasts derived from Axin $1^{\text {Osx }}$ mice compared with BMM cells cultured with CM derived from WT mice. Thus, the loss of Axin 1 in osteoblast precursor cells caused increased OPG and the decrease in osteoclast formation, leading to delayed bone growth in postnatal Axin $1^{\text {Osx }} \mathrm{KO}$ mice.
\end{abstract}

Bone Research (2020)8:31

; https://doi.org/10.1038/s41413-020-0104-5

\section{INTRODUCTION}

$\beta$-catenin is a central molecule in the canonical wingless/ integrated (Wnt) pathway. When Wnt ligands interact with Frizzled and low-density lipoprotein receptor-related protein 5 and 6 coreceptors, $\beta$-catenin is activated, accumulated in the cytoplasm, and translocated into the nucleus. In the nucleus, $\beta$-catenin activates transcription of downstream genes. $\beta$-catenin signaling plays important roles in bone development, postnatal bone growth, and bone remodeling. Animal studies, in which the $\beta$-catenin signaling was either inhibited or activated in chondrocytes, osteoblasts, or osteocytes, demonstrated that activation of $\beta$-catenin signaling promotes osteoblast differentiation and bone formation and inhibition of osteoclast formation and bone resorption. ${ }^{1-7}$ However, the detailed mechanisms of how postnatal bone growth and bone remodeling are regulated by $\beta$-catenin signaling remain unclear.

The $\beta$-catenin signaling in mesenchymal stem cells (MSCs) promoted osteoblast differentiation, inhibited chondrocyte differentiation, and enhanced endochondral ossification. ${ }^{8,9}$ For example, deletion of LRP6, the co-receptor of Wnts, in nestin-expressing cells caused bone mass decrease. ${ }^{10}$ In addition, $\beta$-catenin signaling in mature osteoblasts inhibits osteoclast formation. ${ }^{11-13}$ Activation of $\beta$-catenin signaling also prevented osteoblast apoptosis. ${ }^{14}$ In contrast, the function of $\beta$-catenin signaling in osteoblast precursor cells has not been fully investigated.

Without Wnt ligands interacting with the cell surface receptors, cytoplasmic $\beta$-catenin is degraded by the ubiquitin-proteasome system, mediated by the destruction complex in a phosphorylationdependent manner. Axin 1 and Axin2 are scaffolding proteins in the destruction complex and promote $\beta$-catenin phosphorylation and degradation. Upon Wnt ligand binding to Wnt receptors on the cell surface, the destruction complex is dissociated and $\beta$-catenin is then released from the destruction complex and subsequently translocated into the nucleus.

Several in vivo studies were conducted to determine the role of the $\beta$-catenin destruction complex in bone development. ${ }^{15-17}$ For example, Axin2 KO mice showed craniosynostosis and significantly increased trabecular bone mass. ${ }^{18,19}$ Mice lacking APC, a member in destruction complex, in osteoblasts exhibited dramatically increased bone deposition. ${ }^{11}$ Axin 1 is also a scaffold protein in the destruction complex and is the negative regulator of $\beta$-catenin signaling. ${ }^{15,17}$ Axin1 was expressed ubiquitously, and the systematic deletion of Axin 1 led to early embryonic lethality in mice. ${ }^{20}$ Therefore, exact functions of Axin1 at different differentiation stages during MSC

\footnotetext{
${ }^{1}$ Longhua Hospital, Shanghai University of Traditional Chinese Medicine, 725 WanPing South Road, Shanghai 200032, China; ${ }^{2}$ Spine Institute, Shanghai Academy of Traditional Chinese Medicine, 725 WanPing South Road, Shanghai 200032, China; ${ }^{3}$ Key Laboratory, Ministry of Education of China, 725 WanPing South Road, Shanghai 200032, China; ${ }^{4}$ Research Center for Human Tissues and Organs Degeneration, Shenzhen Institutes of Advanced Technology, Chinese Academy of Sciences, Shenzhen 518055, China; ${ }^{5}$ Department of Orthopedic Surgery, Rush University Medical Center, Chicago, IL 60612, USA and ${ }^{6}$ School of Medicine, Southern University of Science and Technology, Shenzhen 518055, China
}

Correspondence: Yongjun Wang (yjwang88@hotmail.com) or Di Chen (dichen1001@163.com)

These authors contributed equally: Bing Shu, Yongjian Zhao

Received: 26 February 2020 Accepted: 5 March 2020

Published online: 12 August 2020 
differentiation have not been investigated due to the limitation of the lethality of conventional deletion of Axin1.

To determine the potential role of Axin 1 in osteoblast precursor cells at postnatal stage during bone remodeling, we generated Axin $1^{\text {flox flox }}$ mice $^{21}$ and bred these mice with Osx-Cre mice to produce Axin $1^{\text {Osx }}$ conditional KO mice. We found that loss of Axin 1 in osteoblast precursor cells mainly affects osteoclast formation in metaphyseal bone area.

\section{RESULTS}

Increased expression of $\beta$-catenin in $A x i n 1^{\text {Osx }} \mathrm{KO}$ mice

To delete Axin 1 in osteoblasts, primary osteoblasts isolated from calvariae of Axin $7^{\text {flox/flox }}$ mice were infected with adenovirus-Cre recombinase. We found that Axin1 mRNA and protein expressions were significantly decreased in calvarial osteoblasts isolated from Axin $1^{\text {flox/flox }}$ mice infected with Adeno-Cre (Fig. 1a, b). In contrast, $\beta$-catenin expression was increased in these cells (Fig. 1b). We then bred Axin $1^{\text {flox/flox }}$ mice with Osx-Cre mice and generated Axin $1^{\text {Osx }} \mathrm{KO}$ mice. We found that Axin 1 expression was decreased while $\beta$-catenin expression was significantly increased in trabecular bone of tibiae of Axin $1^{\text {Osx }} \mathrm{KO}$ mice (Fig. 1c). In addition, we detected Axin 1 and $\beta$-catenin expression in adipocytes and perivascular cells in the bone marrow. We found that Axin1 expression was decreased while $\beta$-catenin expression was significantly increased in adipocytes (Fig. 1d). However, we did not observe obvious changes in Axin 1 and $\beta$-catenin expression in perivascular cells (Fig. 1d). We also examined changes in Wnt

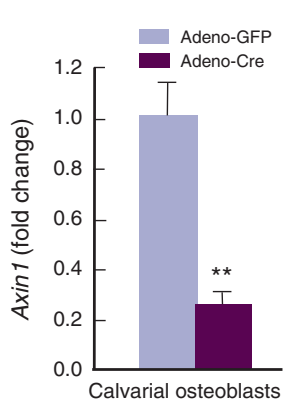

b

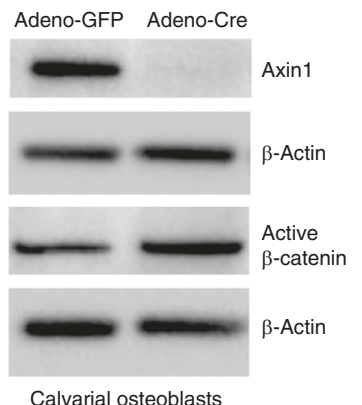

C

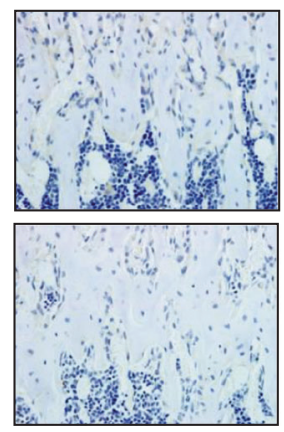

Cre

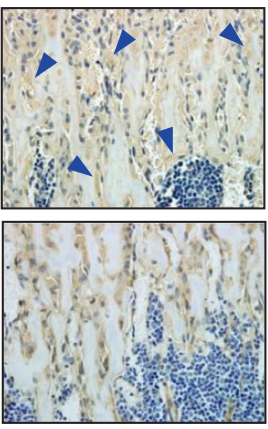

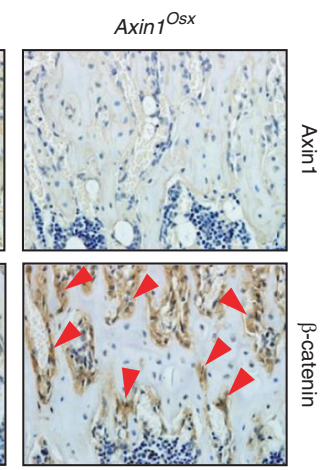

g

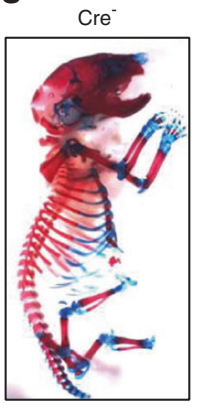

Axin1 ${ }^{\text {Osx }}$

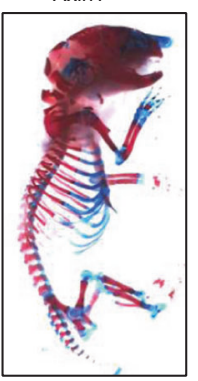

d $\quad \mathrm{Cr}$

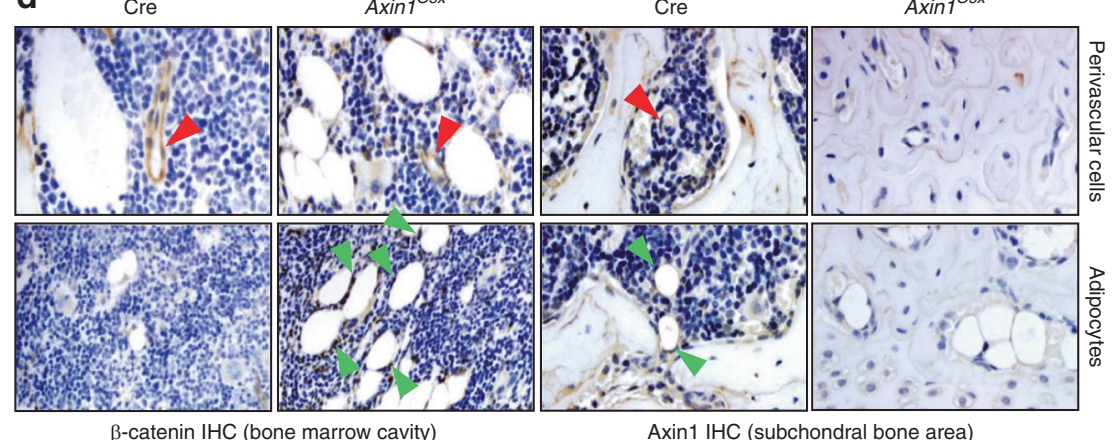

e

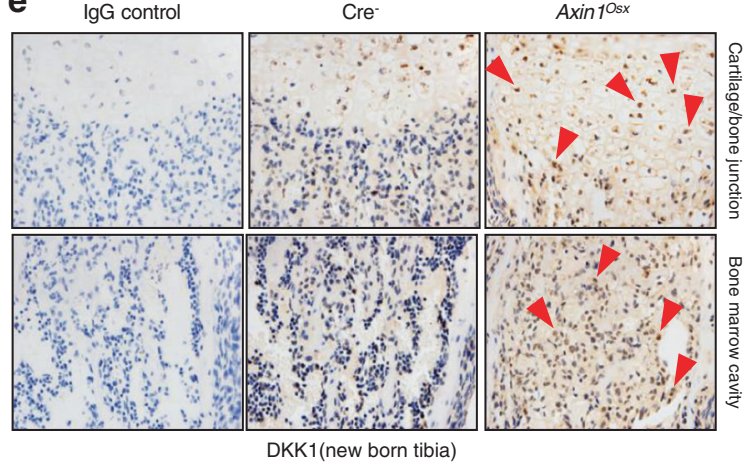

f

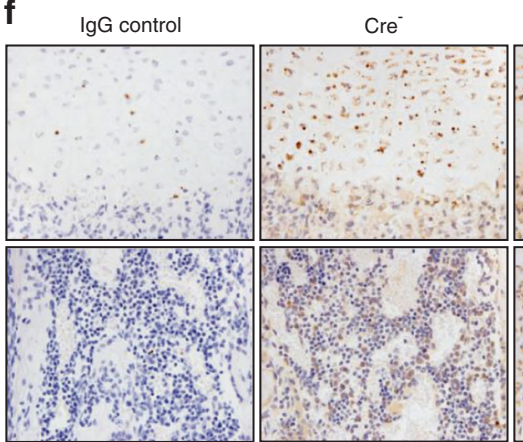

Sclerostin (new born tibia)

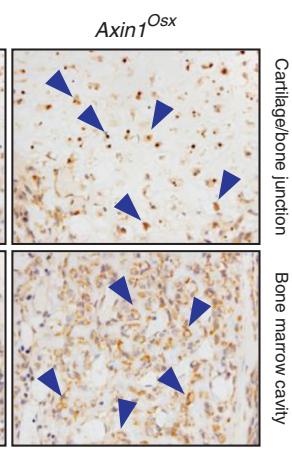

Fig. 1 Increased $\beta$-catenin expression in Axin $1^{\text {Osx }}$ (KO) mice. a Axin 1 expression was significantly decreased in Axin $1^{\text {flox/flox }}$ calvarial osteoblasts infected with Adeno-Cre $\left(n=3,{ }^{*} P<0.01\right)$. b In Axin $1^{\text {flox flox }}$ calvarial osteoblasts infected with Adeno-Cre, Axin 1 protein expression was significantly decreased while active $\beta$-catenin levels were increased. c Results of IHC showed that Axin1 expression (blue arrowheads) was significantly decreased and $\beta$-catenin expression (red arrowheads) was significantly increased on the surface of trabecular bone of newborn Axin $1^{\text {Osx }} \mathrm{KO}$ mice. d Expression of Axin 1 and $\beta$-catenin in perivascular cells (red arrowheads) and in adipocytes (green arrowheads) in bone marrow was analyzed by IHC method. e, f Expression of DKK1 (red arrowheads) and Sclerostin (blue arrowheads) in trabecular bone was analyzed by IHC method. g Newborn Axin $1^{O s x} \mathrm{KO}$ mice and Cre-negative littermates were collected and whole skeletal Alizarin red/Alcian blue staining was performed. No significant changes in skeletal structure in $A x \sin 1^{\text {Osx }} \mathrm{KO}$ mice are seen 

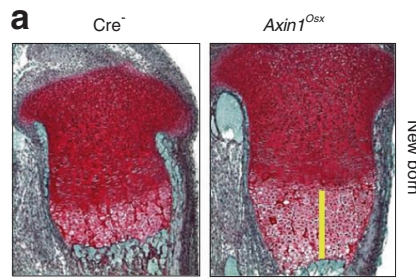

b
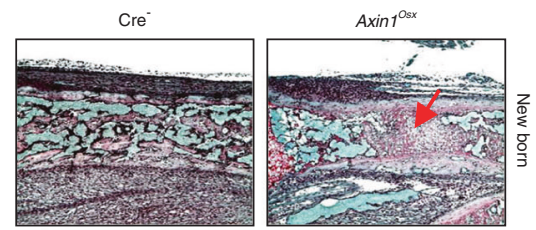

e
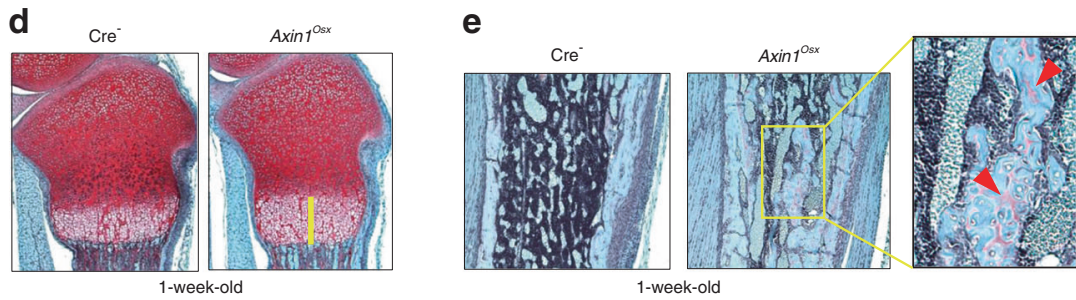

h

g

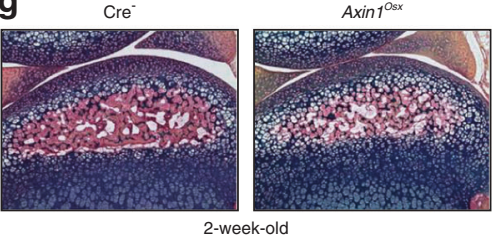

$\operatorname{Axin} 1^{\mathrm{os} x}$

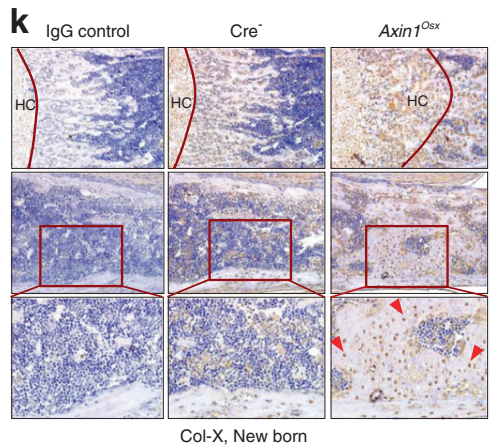

C
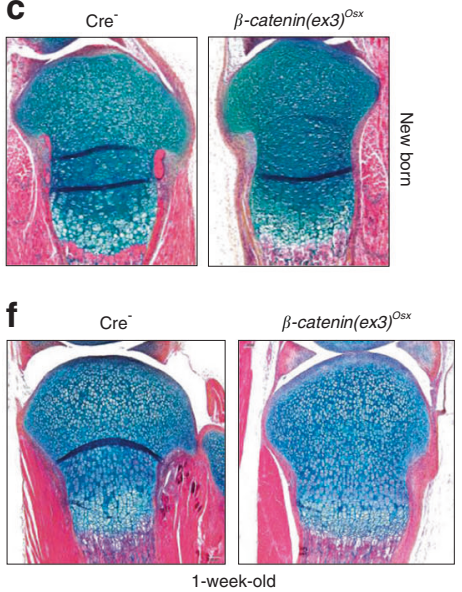

i
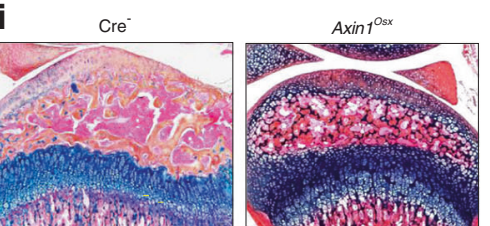

j

Cre

$\beta$-catenin $(e x 3)^{\text {os } x}$

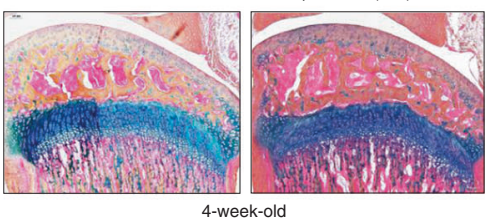

Fig. 2 Delayed postnatal bone growth in $A x i n 1^{\text {Osx }} \mathrm{KO}$ mice. a, d Results of Safranin O/Fast green staining revealed an expanded hypertrophic zone (yellow bars) in tibial growth plates of newborn and 1-week-old Axin $1^{\mathrm{OsX}} \mathrm{KO}$ mice compared with those of Cre-negative littermates. b Results of Alcian blue/Hematoxylin Orange $G(A B / H O)$ staining showed that formation of a primary ossification center was delayed in newborn Axin $1^{\text {Osx }} \mathrm{KO}$ mice (red arrow). e Results of Alcian blue staining showed that trabecular bone with large amounts of uncalcified osteoid (red arrowheads) was found in 1-week-old Axin $1^{\text {Osx }} \mathrm{KO}$ mice. $\mathbf{c}$, $\mathbf{f}$ No significant changes in the length and morphology of growth plate cartilage were seen in newborn and 1-week-old $\beta$-catenin(ex3) ${ }^{\text {Osx }}$ activation mice compared with their Cre-negative littermates. $\mathbf{g}$, $\mathbf{h}$ Results of Alcian blue staining showed a slightly delayed formation of a secondary ossification center in 2 -week-old Axin $1^{\text {Osx }}$ KO mice. In contrast, a significantly delayed formation of a secondary ossification center (red arrow) was found in 2 -week-old $\beta$-catenin(ex3) ${ }^{\text {osx }}$ activation mice. $\mathbf{i}, \mathbf{j}$ Results of Alcian blue staining histology showed relatively normal hypertrophic cartilage development in 4-week-old Axin ${ }^{\text {osx }} \mathrm{KO}$ mice and in 4-week-old $\beta$-catenin (ex3) ${ }^{\text {sx }}$ activation mice. $\mathbf{k}$, I Results of IHC showed that collagen type X (Col-X)-positive hypertrophic chondrocytes (HC) (red arrowheads) and MMP13-positive hypertrophic chondrocytes (blue arrowheads) were found in the metaphyseal bone area and in the middle of the bone marrow cavity of newborn Axin $1^{\text {Osx }}$ KO tibiae

inhibitors by determining the expression of Dkk1 and sclerostin in trabecular bone and found that expression of both Dkk1 and sclerostin was upregulated in trabecular bone area below the growth plate in Axin $1^{\text {Osx }} \mathrm{KO}$ mice (Fig. 1e, f). To determine changes in skeletal structure, newborn Axin $1^{\text {Osx }} \mathrm{KO}$ mice and their littermate controls were collected and performed whole body Alizarin red/Alcian blue staining. We did not observe significant changes in skeletal structure in Axin $1^{\text {Osx }}$ KO mice (Fig. 1g). In contrast, slightly delayed mineralization of calvarial bone was observed in newborn and 4-week-old Axin $1^{\text {Osx }} \mathrm{KO}$ mice (Fig. S1).

Delayed endochondral bone growth in Axin $1^{\text {Osx }} \mathrm{KO}$ mice We performed histological analyses and observed an expanded hypertrophic zone in tibial growth plates of newborn and 1-weekold Axin $1^{\text {Osx }} \mathrm{KO}$ mice (Fig. 2a, d). In newborn Axin $1^{\text {Osx }} \mathrm{KO}$ mice, the length of the hypertrophic zone is almost three times longer than that of Cre-negative mice (Fig. 2a). New bone formation in the primary ossification center was delayed in newborn Axin $1^{\text {Osx }} \mathrm{KO}$ mice (Fig. 2b). In 1 -week-old Axin $1^{\text {Osx }} \mathrm{KO}$ mice, the length of the hypertrophic zone of $A x \operatorname{in} 1^{\text {Osx }} \mathrm{KO}$ mice was significantly longer compared with that of Cre-negative controls (Fig. 2d). Large amounts of uncalcified bone with accumulated osteoid were found in the trabecular bone area below the growth plate (Fig. 2e), suggesting defects in bone remodeling in Axin $1^{\text {Osx }} \mathrm{KO}$ mice. To determine if these changes are due to activation of $\beta$-catenin signaling and to compare the difference between Axin $1^{\text {Osx }} \mathrm{KO}$ mice and $\beta$-catenin conditional activation mice, we generated and analyzed newborn and 1-week-old $\beta$-catenin(ex3) ${ }^{\text {Osx }}$ activation mice. Compared with Axin $1^{\text {Osx }}$ KO mice, we did not observe obvious expansion of hypertrophic cartilage in $\beta$-catenin(ex3) $)^{\text {sx }}$ activation mice (Fig. 2c, f). These findings suggest that Axin 1 may also act through a $\beta$-catenin-independent mechanism to regulate 
a
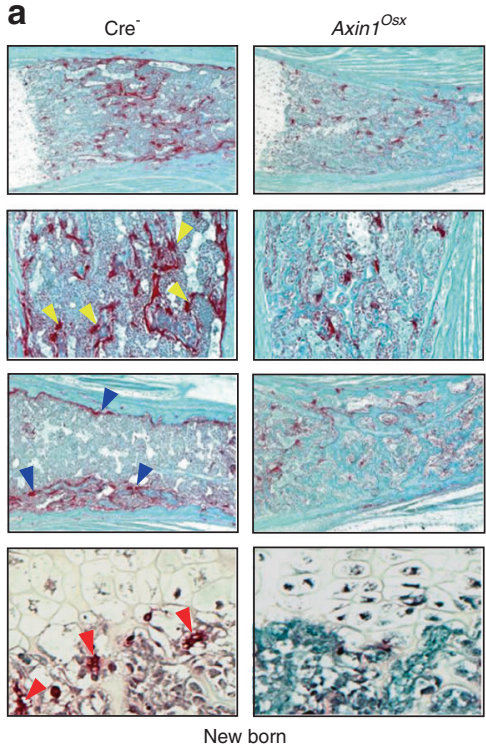

b
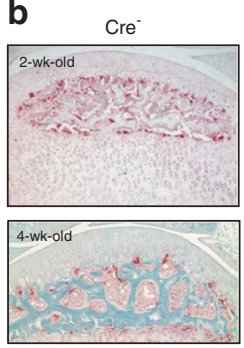
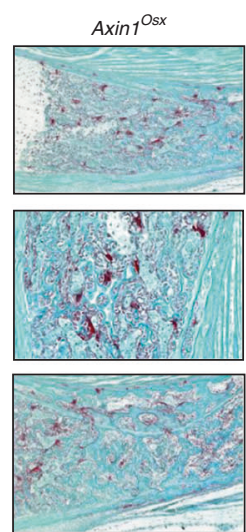

New born
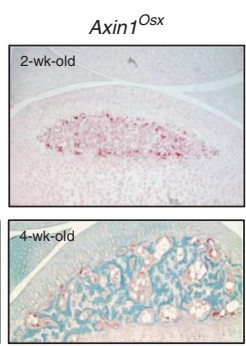

C
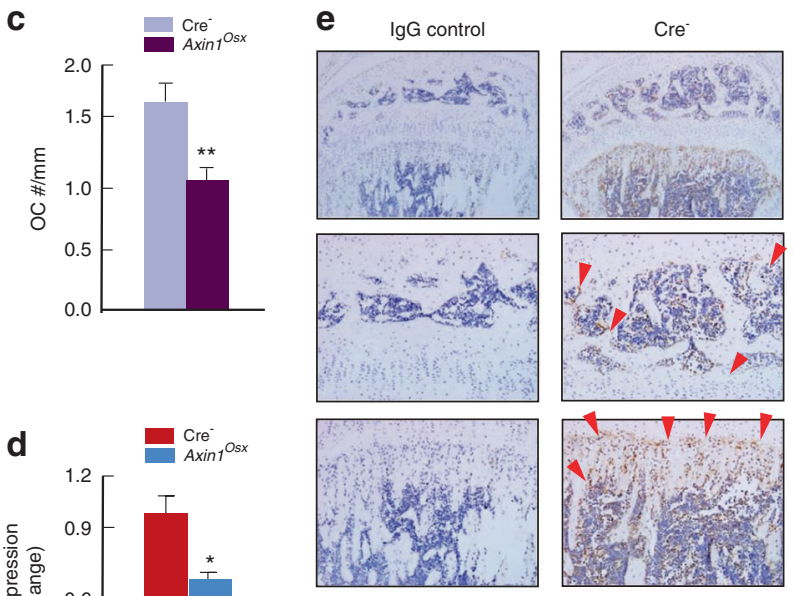

MMP9, 4-week-old mice

g IgG control

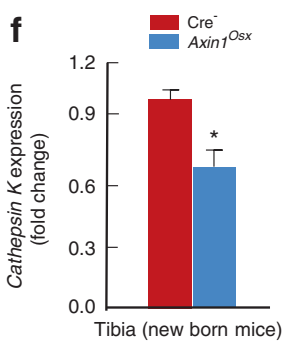

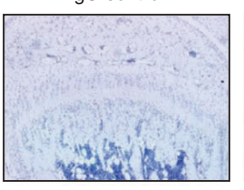

Cre-
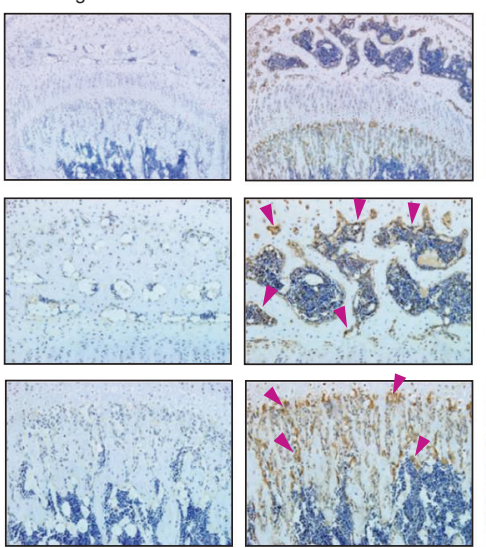
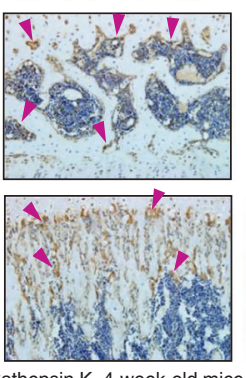
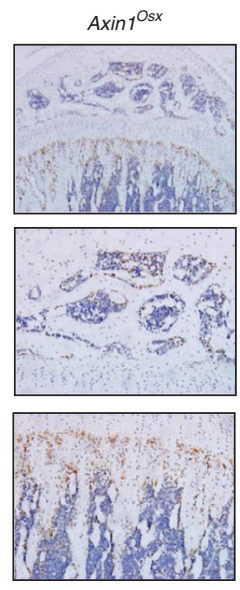

Axin1 ${ }^{\text {Osx }}$
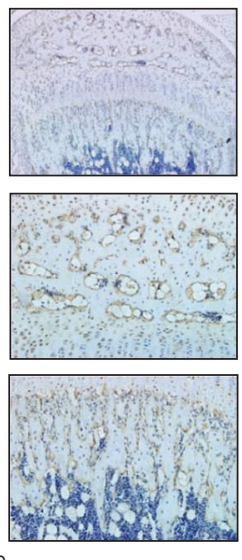

Fig. 3 Decreased osteoclast formation in Axin $1^{\text {Osx }}$ KO mice. a In newborn Cre-negative tibiae, TRAP-positive osteoclasts were found on metaphyseal bone surfaces (yellow arrowheads), the inner region of cortical bone (blue arrowheads) and the ossification front (red arrowheads). In newborn Axin $1^{\text {Osx }}$ KO tibiae, TRAP-positive osteoclasts in the bone marrow cavity and ossification front were significantly decreased. b TRAP-positive osteoclasts were decreased in the subchondral bone area of 2- and 4-week-old Axin ${ }^{\text {osx }}$ KO tibiae. c Osteoclast numbers were quantified. The results showed significantly reduced osteoclast numbers, normalized to trabecular bone perimeters, in the subchondral bone of 4-week-old Axin $1^{\text {Osx }}$ KO mice compared with those of Cre-negative mice $(n=4, * * P<0.01)$. d-g Real-time PCR and immunohistochemistry (IHC) data showed decreased mRNA and protein expressions of MMP9 (red arrowheads) and cathepsin K (purple arrowheads) in subchondral bone (middle panel) and metaphyseal bone (lower panel) of 4-week-old $A x i n 1^{\text {Osx }} \mathrm{KO}$ tibiae. ( $n=4$, ${ }^{*} P<0.05$ )

postnatal bone growth. In 2-week-old $\operatorname{Axin} 1^{\text {Osx }}$ KO mice, we observed a slightly delayed formation of a secondary ossification center (Fig. 2g). In contrast, the formation of secondary ossification centers were significantly delayed in $\beta$-catenin(ex3) ${ }^{\text {sx }}$ activation mice (Fig. 2h). In 4-week-old mice, it seems that growth plate cartilage development and the formation of a secondary ossification center were normal in Axin $1^{\text {Osx }}$ KO mice (Fig. 2i) or in $\beta$-catenin(ex3) ${ }^{\text {sx }}$ activation mice (Fig. 2j). Results of IHC analyses showed that extensively increased Col-X-positive hypertrophic chondrocytes were found in the metaphyseal bone area of newborn Axin $1^{\text {Osx }}$ KO tibiae (Fig. 2k). Similarly, extensively increased MMP13-positive cells were also found in the expanded hypertrophic zone (Fig. 2l). This phenotype was 100\% penetrated in Axin $1^{\text {Osx }} \mathrm{KO}$ mice.

Impaired osteoclast formation in Axin $1^{\text {Osx }} \mathrm{KO}$ mice

Osteoclasts in growth plate can phagocytose dying hypertrophic chondrocytes and absorb the mineralized cartilage matrix. Therefore, we analyzed changes in osteoclast formation in Axin ${ }^{\text {Osx }} \mathrm{KO}$ mice. In newborn Cre-negative tibiae, large numbers of TRAPpositive osteoclasts were found on the metaphyseal bone area and the inner side of cortical bone (Fig. 3a). In addition, osteoclasts were also found in the ossification front, where osteoclasts invaded hypertrophic chondrocytes (Fig. 3a, lower left panel). In newborn Axin $1^{\text {Osx }} \mathrm{KO}$ tibiae, TRAP-positive osteoclasts in the bone marrow cavity and ossification front were significantly decreased (Fig. 3a, right panel). Osteoclast formation in the subchondral bone of 2- and 4-week-old Axin $1^{\text {Osx }}$ KO tibiae was also decreased compared with that of Cre-negative tibiae (Fig. 3b). Quantification of the ratios of osteoclast numbers and trabecular bone perimeters showed reduced TRAP-positive osteoclast numbers in 4-week-old Axin $1^{\text {Osx }}$ KO mice (Fig. 3c). Real-time PCR and IHC results also showed decreased expression of osteoclast markers, MMP9 (Fig. 3d, e) and cathpesin K (Fig. 3f, g) in both subchondral bone and metaphyseal bone of 4 -week-old Axin $1^{\text {Osx }} \mathrm{KO}$ tibiae. By contrast, no obvious changes in osteoblast numbers were found in the metaphyseal bone of same-aged Axin $1^{\text {Osx }} \mathrm{KO}$ mice (Fig. 4a, b). Results of calcein labeling and quantification of mineral appositional rates (MAR) showed that bone formation of cortical bone was not significantly changed in Axin $1^{\text {Osx }} \mathrm{KO}$ mice (Fig. 4c, d).

Increased Opg expression in Axin $1^{\text {Osx }}$ KO mice

The ratio of Opg/Rankl is an important index reflecting changes in osteoclast formation. In the tibiae of Cre-negative mice, osteoprotegerin (OPG)-positive staining cells were observed on the trabecular bone surface (Fig. 5a). In Axin $1^{\text {Osx }} \mathrm{KO}$ mice, the number 
a
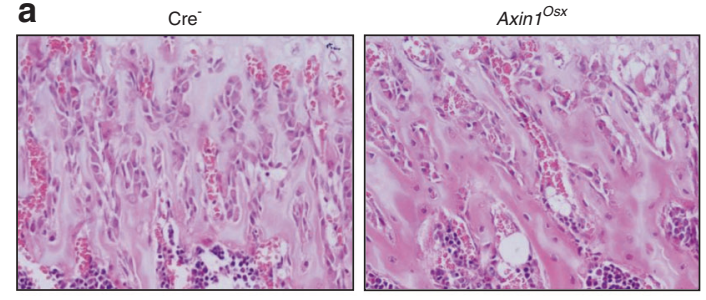

C
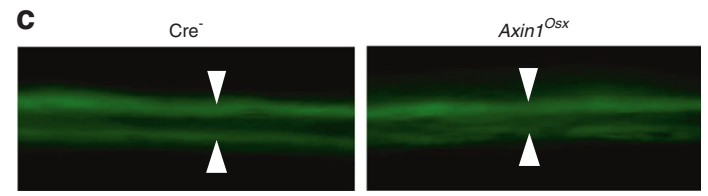

b
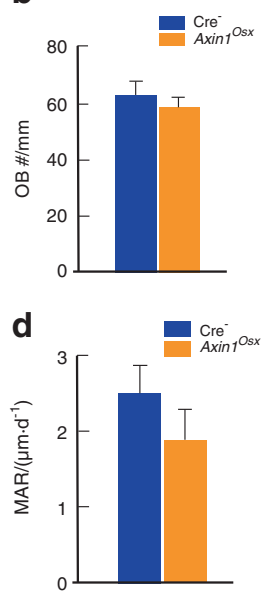

Fig. 4 No significant change in the bone formation in Axin $1^{\text {Osx }} \mathrm{KO}$ mice. a Hematoxylin and eosin (HE) staining of trabecular bone in the metaphyseal area of 4-week-old Axin ${ }^{\text {Osx }} \mathrm{KO}$ mice and Cre-negative controls. b Quantification of osteoblast numbers showed no significant changes in osteoblast numbers in trabecular bone in the metaphyseal area of 4-week-old $A x i n 1^{\text {Osx }} \mathrm{KO}$ mice $(n=6)$. c, $\mathbf{d}$ Results of calcein labeling and the measurement of mineral appositional rates (MAR) showed cortical bone formation was slightly reduced in 4 -week-old Axin $1^{\text {Sx }}$ KO mice $(n=6)$

and staining intensity of OPG-positive cells were significantly increased in Axin $1^{\text {Osx }} \mathrm{KO}$ mice, especially on the trabecular bone surface (Fig. 5a). Osteoblasts are the important cell resource for OPG production and osteoblast precursor cells are the Osterixexpressing cells. Therefore, we examined changes in Opg and Rankl expression in primary calvarial osteoblasts of newborn Axin $1^{\text {Osx }} \mathrm{KO}$ mice and Cre-negative littermate controls. In calvarial osteoblasts of newborn Axin $1^{\text {Osx }}$ KO mice, though both Opg and Rankl expressions were increased (Fig. 5b, c), the Opg:Rankl ratio was still significantly higher in Axin1-deficient osteoblasts than that of control osteoblasts (Fig. $5 \mathrm{~d}$ ). We then cultured wild-type (WT) bone marrow derived macrophages (BMM) cells treated with the conditioned media (CM) collected from primary osteoblasts isolated from Axin $1^{\text {Osx }} \mathrm{KO}$ mice and Cre-negative mice, respectively. Osteoclast formation in the BMM cells treated with CM collected from osteoblasts of Axin $1^{\text {Osx }} \mathrm{KO}$ mice was obviously decreased compared with the cells treated with CM collected from control osteoblasts (Fig. 5e). These findings were further confirmed by the results of osteoclast quantification (Fig. 5f). Nuclear factor of activated T-cells, cytoplasmic 1 (NFATc-1) and cFos are two key regulators of osteoclast differentiation upon receptor activator of nuclear factor kappa-B ligand (RANKL) induction. Real-time PCR assay revealed that expression of Nfatc1 and c-Fos was decreased in trabecular bone of Axin $1^{\text {Osx }}$ KO mice (Fig. $5 \mathrm{~g}, \mathrm{~h}$ ). IHC assays further demonstrated that NFATc- $1-$ and cFos-expressing cells were detected in trabecular bone, and that NFATc- 1 was especially highly expressed in the ossification front of the tibiae in control mice (Fig. $5 \mathrm{i}, \mathrm{j}$ ). The numbers of NFATc- 1 and c-Fos positive cells were significantly decreased in the tibiae of Axin $1^{\text {Osx }} \mathrm{KO}$ mice (Fig. $5 \mathrm{i}, \mathrm{j}$ ).

\section{DISCUSSION}

Osterix is the osteoblast specific transcription factor that regulates osteoblast precursor cell differentiation and inhibits cell proliferation. ${ }^{22,23}$ In Osx-expressing osteoblast precursor cells, Wnt/ $\beta$-catenin signaling activation is at a low level. Osx-Cre targeting cells were established by breeding Osx-Cre mice with $R O S A^{m T / m G}$ reporter mice, followed by fluorescence microscopic analysis. Osx-Cre targeting cells were detected in trabecular bone and on the endosteal region of cortical bone; they were especially highly expressed in the area of metaphyseal bone that is close to the growth plate hypertrophic cartilage area. ${ }^{22}$ Consistent with these findings, when Axin1 expression was inhibited in osteoblast precursor cells using Osx-Cre transgenic mice, $\beta$-catenin-positive staining cells were also detected in trabecular bone and on the endosteal region of the cortical bone.

In this project we discovered that deletion of Axin 1 in osteoblast precursor cells led to $\beta$-catenin upregulation, which further resulted in increased OPG expression. Increased OPG expression could inhibit osteoclast formation, which may be responsible for the reduction of apoptosis in hypertrophic chondrocytes and decreased resorption of mineralized cartilage matrix. Wnt/ $\beta$-catenin signaling controls osteoblast and osteoclast differentiation and regulates bone mass. ${ }^{24,25}$ In our study, osteoblast numbers and bone mass were not significantly changed in Axin $1^{\text {Osx }}$ KO mice. In contrast, osteoclast formation was dramatically decreased when Axin 1 was deleted in Osxexpressing osteoblast precursor cells. In Axin $1^{\text {Osx }}$ KO mice, $\beta$-catenin levels were increased in osteoblast precursor cells. It is known that inhibition of $\beta$-catenin signaling causes an increase in chondrocyte apoptosis during postnatal cartilage development. ${ }^{26} \beta$-catenin, together with TCF proteins, regulates Opg expression in osteoblasts. ${ }^{12}$ Loss of $\beta$-catenin in mature osteoblasts resulted in a decreased ratio of OPG/RANKL, while accumulation of $\beta$-catenin signaling led to an increased ratio of OPG/RANKL. ${ }^{11-13}$ OPG is a decoy receptor that prevents RANKL/ RANK interaction and inhibits osteoclast differentiation. ${ }^{27-29}$ Therefore, activation of $\beta$-catenin signaling in mature osteoblasts resulted in a decrease in osteoclast formation, while inhibition of $\beta$-catenin signaling caused an increase in osteoclast formation leading to severe osteopenia. ${ }^{11-13}$ In addition, $\beta$-catenin signaling in chondrocytes could also regulate osteoclast formation and bone resorption through regulation of OPG expression. ${ }^{30}$ In this report, we have demonstrated that deletion of Axin1 in osteoblast precursor cells led to upregulation of $\beta$-catenin signaling, increased the ratio of OPG/RANKL and prevented osteoclast formation. We also found that Rankl expression in primary calvarial osteoblasts of Axin $1^{\text {Osx }}$ mice was also upregulated. This is not consistent with previous reports that $\beta$-catenin signaling downregulated Rankl expression in a glucocorticoid receptor-dependent manner. ${ }^{27}$ This discrepancy may be due to the possibility that Axin 1 may also affect other signaling molecules in addition to that of $\beta$-catenin. In fact, comparing differences in the histological results of newborn, 1-week-old and 2week-old Axin $1^{\text {Osx }} \mathrm{KO}$ mice with those of $\beta$-catenin $(\text { ex } 3)^{\text {Osx }}$ activation mice, the phenotypes of the expanded hypertrophic zone and formation of the secondary ossification center are very different between Axin $1 \mathrm{KO}$ mice and $\beta$-catenin activation mice, suggesting 
a
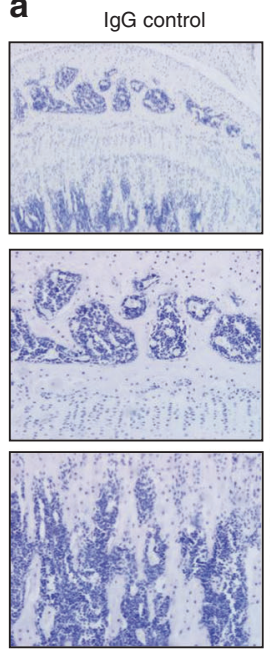

Cre
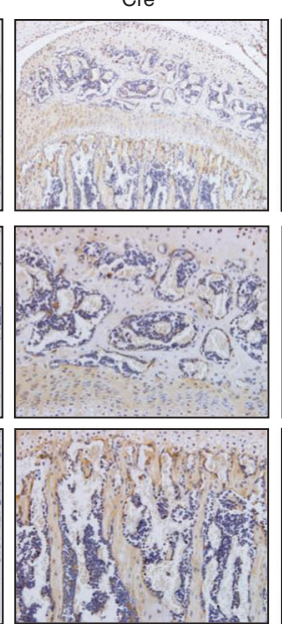

OPG, 4-week-old

i

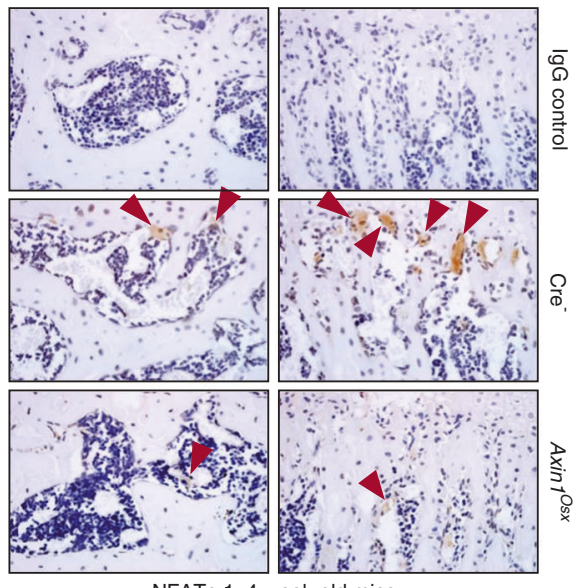

Axin $^{\text {Osx }}$
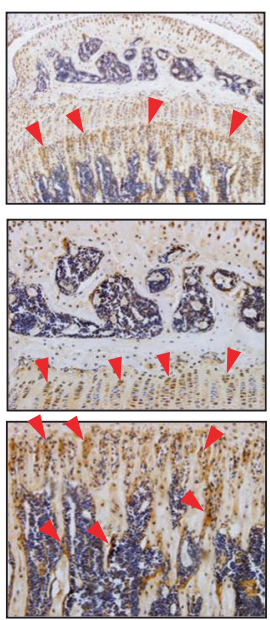

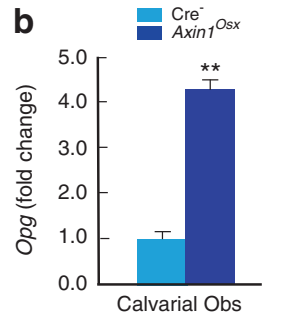

e
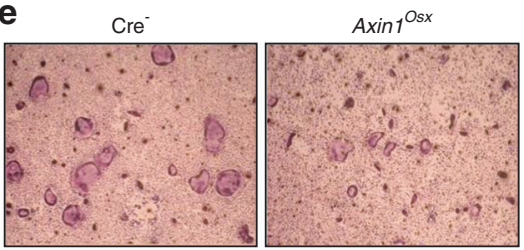
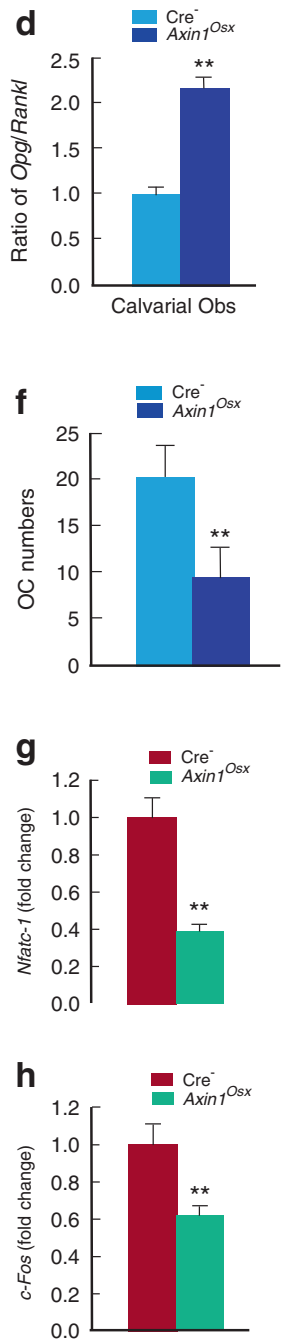

Fig. 5 Alterations of OPG, NFATc-1, and c-Fos expression in Axin $1^{\text {Osx }} \mathrm{KO}$ mice. a Results of IHC staining showed that more osteoprotegerin (OPG)-positive cells were observed in 4-week-old Axin $1^{\text {Osx }} \mathrm{KO}$ mice, especially on the surface of trabecular bone (red arrowheads). b-d In calvarial osteoblasts isolated from newborn Axin $1^{\text {Osx }} \mathrm{KO}$ mice, both Opg and Rankl expression was increased, and the ratio of Opg/Rankl was significantly higher in bone marrow stromal (BMS) cells derived from Axin $1^{\text {Osx }}$ mice than that of Cre-negative mice $\left(n=4,{ }^{* *} P<0.01\right)$. e WT BMM cells were cultured with the conditioned medium (CM) collected from cultured calvarial osteoblasts of 4-week-old Axin $1^{\text {Osx }}$ KO mice and Cre-negative littermates, respectively. Osteoclast formation in the cells cultured with CM from the Axin $1^{\text {Osx }} \mathrm{KO}$ calvarial cells was significantly decreased compared with that in the cells cultured with CM collected from Cre-negative osteoblasts. f Quantification results showed decreased osteoclast numbers in the Axin $1^{\text {Osx }} \mathrm{KO}$ group $\left(n=6,{ }^{* *} P<0.01\right)$. g, $\mathbf{h}$ The total RNA was extracted from tibia of 4 -week-old Crenegative and Axin $1^{\text {Osx }} \mathrm{KO}$ mice and Nfatc- 1 and c-Fos mRNA expression was detected by real-time PCR $\left(n=4,{ }^{* * P}<0.01\right)$. i, $\mathbf{j}$ In tibiae of 4 -weekold Axin $1^{\text {Osx }}$ KO mice, NFATc-1-positive staining cells (brown arrowheads) and c-Fos-positive staining cells (purple arrowheads) were decreased compared with those of Cre-negative control mice

that Axin1 may also act through a $\beta$-catenin-independent mechanism to regulate postnatal bone growth.

During the endochondral bone formation process, hypertrophic chondrocytes in the growth plate undergo apoptosis and the mineralized cartilage matrix was absorbed, followed by bone formation. At the ossification front, hypertrophic chondrocytes directly contact with osteoclasts. Findings with annexin-V labeling confirmed that osteoclasts could remove dying chondrocytes by phagocytosis. ${ }^{31}$ Meanwhile, osteoclasts are also capable of absorbing the mineralized cartilage matrix. ${ }^{32,33}$ Mice lacking TRAP resulted in an expanded hypertrophic zone and disordered hypertrophic chondrocyte columns that extended into the trabecular bone region. ${ }^{34}$ Mice lacking RANK showed an even more severe phenotype of an expanded hypertrophic zone. The bone marrow cavity was almost filled with unabsorbed cartilage. ${ }^{35,36}$ In Axin $^{\text {Osx }} \mathrm{KO}$ mice, we also found an expanded hypertrophic zone and unabsorbed cartilage in the bone marrow cavity, which may be caused by decreased osteoclast formation. Meanwhile, large numbers of Col-X-positive prehypertrophic and hypertrophic chondrocytes and MMP13-expressing terminally differentiated hypertrophic chondrocytes were found in the expanded hypertrophic zone of the growth plate as well as in the bone marrow cavity. This suggests that the terminal apoptosis of chondrocytes was inhibited or delayed due to the loss of Axin 1 in osteoblast precursor cells. Although Osterix was also detected in a subset of chondrocytes, ${ }^{37,38}$ in Axin $1^{\text {Osx }} \mathrm{KO}$ mice, $\beta$-catenin upregulation was found in the chondrocytes located in the upper part of the proliferative zone, but not in the hypertrophic zone. However, the phenotypes of the expanded hypertrophic zone and unabsorbed cartilage matrix observed in Axin $1^{\text {Osx }} \mathrm{KO}$ mice may not be primarily caused by the alteration of $\beta$-catenin signaling in hypertrophic chondrocytes. The evidence that the phenotype of 
Inhibition of osteoclast formation in $A x \sin 1^{\text {Osx }}$ KO mice

B Shu et al.

the expanded hypertrophic zone was not observed in $\beta$-catenin activation mice further suggest that the phenotype observed in early postnatal Axin $1 \mathrm{KO}$ mice was not caused by the activation of $\beta$-catenin signaling. The detailed mechanism of Axin1 regulation of postnatal bone growth needs to be further investigated.

In conclusion, loss of Axin1 in osteoblast precursor cells led to activation of $\beta$-catenin signaling, which in turn upregulated OPG expression and increased the ratio of OPG/RANKL. Osteoclast formation was then inhibited by OPG, which is responsible for decreased apoptosis of hypertrophic chondrocytes and reduced resorption of the mineralized cartilage matrix. However, it is not totally clear whether only a small portion of osteoblast precursor cells was affected leading to the changes in $\beta$-catenin signaling in those cells. This possibility needs to be further investigated.

\section{MATERIALS AND METHODS}

Animals

The use of animals was approved by Shanghai Laboratory Animal Use Committee. The generation of Axin $1^{\text {flox/flox }}$ mice has been reported in previous studies. ${ }^{21}$ Osx-Cre mice were obtained from the Jackson Laboratory. Axin $1^{\text {Osx }}$ conditional KO mice were generated by crossing Axin $1^{\text {flox/flox }}$ mice with Osx-Cre transgenic mice (Osx-Cre;Axin $\left.1^{\text {flox/flox }}\right)$. $\beta$-catenin $(e x 3)^{\text {flox/- }}$ mice have been used in our previous studies. ${ }^{30,39}$

Isolation and culture of calvarial osteoblasts

The periosteal layers on both sides of the skull of 3-day-old mice were removed. The calvariae were transferred into a $50 \mathrm{~mL}$ conical tube and digested in $1 \mathrm{mg} \cdot \mathrm{mL}^{-1}$ collagenase $A$ (Roche, Basel, Switzerland) in serum-free aMEM in a $37^{\circ} \mathrm{C}$ water bath for $15 \mathrm{~min}$. After two repeated digestions in fresh collagenase $A$ solution, the retained mixtures of collagenase $A$ and cells were filtered into a new tube. The cell suspensions were mixed, centrifuged, and resuspended in aMEM with $10 \% \mathrm{FBS}$ and $50 \mu \mathrm{g} \cdot \mathrm{mL}^{-1}$ ascorbic acid. The suspension was finally plated in six-well culture plates at a density of $2 \times 10^{5}$ cells per well. The media were changed every other day.

Adenovirus mediated deletion of Axin 1 in primary calvarial osteoblasts

Primary osteoblasts were isolated from calvariae of Axin $1^{\text {flox/flox }}$ mice and cultured in aMEM with $10 \%$ FBS in six-well plates. When cells reach $40 \%$ density, Adeno-Cre or Adeno-GFP (Hanbio, Shanghai, China) was added to the culture medium at a concentration of $2 \times$ $10^{8}$ plaque-forming unit (PFU) per $\mathrm{mL}$. The culture medium was changed $24 \mathrm{~h}$ after infection. The cells were collected $40 \mathrm{~h}$ after virus infection for real-time PCR and western blot assays.

Real-time PCR assay

Total RNA was extracted from primary calvarial osteoblasts using a RNeasy mini kit (Qiagen, Hilden, Germany). DNAse I-treated total RNA was reverse transcripted using a RT reagent kit (Takara Bio, Tokyo, Japan). The cDNA was amplified by PCR in a total volume of $20 \mu \mathrm{L}$ reaction solution containing $10 \mathrm{pmol} \cdot \mathrm{L}^{-1}$ primers (primer names and sequences are listed in Table 1).

Whole skeleton Alizarin red and Alcian blue staining

After removing skin and adipose tissues, the skeletons were fixed in $95 \%$ ethanol for 2 days followed by fixation in acetone for another day. The skeletons were then stained with $0.015 \%$ Alcian blue and $0.005 \%$ Alizarin red for 3 days. Pictures were taken after most of the soft tissue was digested in $1 \%$ potassium chloride solution.

Histological analysis

Tibial samples were fixed in $4 \%$ paraformaldehyde, decalcified, dehydrated, and embedded in paraffin. 4- $\mu \mathrm{m}$ thick serial midsagittal sections of tibias were cut and stained with Alcian blue/HEG (ABH) and Safranin O/Fast green (SOF).
Table 1. Names and sequences of PCR primers

\begin{tabular}{ll}
\hline Gene Name & Sequence \\
\hline$\beta$-actin (forward) & $5^{\prime}$-GGAGATTACTGCCCTGGCTCCTA-3' \\
$\beta$-actin (reverse) & $5^{\prime}$-GACTCATCGTACTCTGCTTGCTG-3' \\
Axin1 (forward) & $5^{\prime}$-GGACCTCGGAGCAAGTTTCA-3' \\
Axin1 (reverse) & $5^{\prime}$-GGTTGACAGGCCTCGAATCA-3' \\
Opg (forward) & $5^{\prime}$-CAGAGCGAAACAC AGTTTG-3' \\
Opg (reverse) & $5^{\prime}$-CACACAGGGTGACATCTATTC-3' \\
Rankl (forward) & $5^{\prime}$-CAGGTTTGCAGGACTCGAC-3' \\
Rankl (reverse) & $5^{\prime}$-AGCAGGGAAGGGTTGGACA-3' \\
Mmp9 (forward) & $5^{\prime}$-CCATGCACTGGGCTTAGATCA-3' \\
Mmp9 (reverse) & $5^{\prime}$-GGCCTTGGGTCAGGCTTAGA-3' \\
Cathepsin K (forward) & $5^{\prime}$-CAGCAGAACGGAGGCATTGA-3' \\
Cathepsin K (reverse) & $5^{\prime}$-CTTTGCCGTGGCGTTATACATACA-3' \\
NFATc-1 (forward) & $5^{\prime}$-CCGTTGCTTCCAGAAAATAACA-3' \\
NFATc-1 (reverse) & $5^{\prime}$-TGTGGGATGTGAACTCGGAA-3' \\
c-Fos (forward) & $5^{\prime}$-CGGGTTTCAACGCCGACTAC-3' \\
c-Fos (reverse) & $5^{\prime}$-AAAGTTGGCACTAGAGACGGACAGA-3' \\
\hline
\end{tabular}

Histomorphometric analysis was performed using an Olympus BX50 microscope (Olympus, Tokyo, Japan) and Image-Pro Express software (Media Cybernetics, Rockville, MD, USA). TRAP-positive osteoclast numbers were quantified.

\section{Calcein labeling assay}

$1 \mathrm{mg} \cdot \mathrm{mL}^{-1}$ calcein in saline was injected into 3-week-old mice (i.p. injection, $10 \mathrm{mg} \cdot \mathrm{kg}^{-1}$ ) followed by a second injection 4 days later. Tibiae of 4-week-old mice were fixed in $4 \%$ paraformaldehyde and dehydrated in a series of ethanol (75\%-100\%). Samples were embedded in methyl methacrylate and $100-\mu \mathrm{m}$ thick serial midsagittal sections were cut. The fluorescence signal of the cortical bone was observed using an Olympus BX50 microscope (Olympus). The distances of two calcein labels were measured with DP Manager software (Olympus) to determine the MAR.

\section{TRAP staining}

Paraffin sections of newborn, 2- and 4-week-old tibias were rehydrated and incubated with a $0.4 \mathrm{mg} \cdot \mathrm{mL}^{-1}$ Napthol-Ether solution/basic stock incubation solution at $37^{\circ} \mathrm{C}$ for $30 \mathrm{~min}$. A $0.04 \mathrm{~g} \cdot \mathrm{mL}^{-1}$ sodium nitrite solution and a $0.05 \mathrm{~g} \cdot \mathrm{mL}^{-1}$ pararosaniline dye/2 $\mathrm{N}$ hydrochloric acid solution was mixed and added to the basic stock incubation solution and the paraffin sections were incubated in this mixture for $10 \mathrm{~min}$.

Immunohistochemistry (IHC) staining

Paraffin sections of 4-week-old tibiae were rehydrated and digested in $0.1 \%$ trypsin for $10 \mathrm{~min}$ at the room temperature, and then treated with $3 \% \mathrm{H}_{2} \mathrm{O}_{2}$ for $20 \mathrm{~min}$. Sections were incubated with primary antibodies in PBS overnight at $4{ }^{\circ} \mathrm{C}$. Col$X$, MMP13, MMP9, cathepsin K, OPG, DKK1, and sclerostin antibodies were obtained from Abcam (Cambridge, MA, USA). Axin1 and $\beta$-catenin antibodies were obtained from Sigma (St. Louis, MO, USA). NFATc-1 and c-Fos antibodies were obtained from Santa Cruz Biotechnology (Santa Cruz, CA, USA). Negative control sections were incubated with IgG (Beyotime Biotechnology, Shanghai, China). A Polink-2 plus polymer HRP detection kit (PV9001, ZSGB-BIO, Shanghai, China) was used for incubation with secondary antibody and horseradish peroxidase (HRP)-streptavidin.

In vitro osteoclast differentiation assay

BMM cells were isolated from long bone of 1-month-old WT mice and plated into 24 -well culture plates at a density of $4 \times 10^{5}$ cells 
per well and cultured in aMEM with 10\% FBS. Cells were treated with $20 \mathrm{ng} \cdot \mathrm{mL}^{-1}$ macrophage colony-stimulating factor (M-CSF) for 3 days, and then switched to the medium with $10 \mathrm{ng} \cdot \mathrm{mL}^{-1} \mathrm{M}$ CSF and $50 \mathrm{ng} \cdot \mathrm{mL}^{-1}$ RANKL for 7 days. To test Axin $1^{\text {Osx }} \mathrm{CM}, \mathrm{BMM}$ cells were treated with $\mathrm{CM}$ collected from cultured calvarial osteoblasts isolated from Axin $1^{\text {Osx }} \mathrm{KO}$ mice and Cre-negative controls for 7 days. The culture medium was changed every 3 days. TRAP staining was performed using a TRAP assay kit (Sigma, St. Louis, MO, USA).

\section{Statistical analysis}

An unpaired Student's $t$ test was performed for experiments involving two groups. $P<0.05$ was considered statistically significant.

\section{DATA AVAILABILITY}

All data and materials used in the analysis are available to any researcher for purposes of reproducing or extending the analysis.

\section{ACKNOWLEDGEMENTS}

This work was supported by the following funding agencies. (1) National Natural Science Foundation of China (NSFC) $(81973876,81673991$ to BS, 81730107 to YJW and 81603643 to YJZ). (2) The National Key R\&D Program of China (2018YFC1704302 to YJW). The Program for Innovative Research Team in University, Ministry of Education of China (IRT1270 to YJW). The Program for Innovative Research Team, Ministry of Science and Technology of China (2015RA4002 to YJW). The Three Years Action to Accelerate the Development of Traditional Chinese Medicine Plan (ZY (2018-2020)-CCCX-3003 to YJW). (3) National Natural Science Foundation of China (NSFC) (81672227) and a Frontier Science of CAS grant (QYZDB-SSW-JSC030) to HP. National Natural Science Foundation of China (NSFC) (81991513) to GX.

\section{ADDITIONAL INFORMATION}

The online version of this article (https://doi.org/10.1038/s41413-020-0104-5) contains supplementary material, which is available to authorized users.

Competing interests: The authors declare no competing interests.

\section{REFERENCES}

1. Tu, X. et al. Osteocytes mediate the anabolic actions of canonical Wnt/betacatenin signaling in bone. Proc. Natl. Acad. Sci. USA 112, E478-E486 (2015).

2. Tamamura, Y. et al. Developmental regulation of $\mathrm{Wnt} /$ beta-catenin signals is required for growth plate assembly, cartilage integrity, and endochondral ossification. J. Biol. Chem. 280, 19185-19195 (2005).

3. Morvan, F. et al. Deletion of a single allele of the Dkk1 gene leads to an increase in bone formation and bone mass. J. Bone Miner. Res 21, 934-945 (2006).

4. Kitagaki, J. et al. Activation of beta-catenin-LEF/TCF signal pathway in chondrocytes stimulates ectopic endochondral ossification. Osteoarthr. Cartil. 11, 36-43 (2003).

5. Kramer, I. et al. Osteocyte Wnt/beta-catenin signaling is required for normal bone homeostasis. Mol. Cell Biol. 30, 3071-3085 (2010).

6. Lu, C. et al. Wnt-mediated reciprocal regulation between cartilage and bone development during endochondral ossification. Bone 53, 566-574 (2013).

7. Burgers, T. A. et al. Mice with a heterozygous Lrp6 deletion have impaired fracture healing. Bone Res. 4, 16025 (2016).

8. Day, T. F. et al. Wnt/beta-catenin signaling in mesenchymal progenitors controls osteoblast and chondrocyte differentiation during vertebrate skeletogenesis. Dev. Cell 8, 739-750 (2005)

9. Hill, T. P. et al. Canonical Wnt/beta-catenin signaling prevents osteoblasts from differentiating into chondrocytes. Dev. Cell 8, 727-738 (2005).

10. Li, C. et al. LRP6 in mesenchymal stem cells is required for bone formation during bone growth and bone remodeling. Bone Res. 2, 14006 (2014).

11. Holmen, S. L. et al. Essential role of beta-catenin in postnatal bone acquisition. J. Biol. Chem. 280, 21162-21168 (2005).

12. Glass, D. A. 2nd et al. Canonical Wnt signaling in differentiated osteoblasts controls osteoclast differentiation. Dev. Cell 8, 751-764 (2005).

13. Glass, D. A. 2nd \& Karsenty, G. Canonical Wnt signaling in osteoblasts is required for osteoclast differentiation. Ann. NY Acad. Sci. 1068, 117-130 (2006).

14. Almeida, M. et al. Wnt proteins prevent apoptosis of both uncommitted osteoblast progenitors and differentiated osteoblasts by beta-catenin-dependent and -independent signaling cascades involving Src/ERK and phosphatidylinositol 3kinase/AKT. J. Biol. Chem. 280, 41342-41351 (2005).

15. Li, V. S. et al. Wnt signaling through inhibition of beta-catenin degradation in an intact Axin1 complex. Cell 149, 1245-1256 (2012).

16. Ha, N. C. et al. Mechanism of phosphorylation-dependent binding of APC to betacatenin and its role in beta-catenin degradation. Mol. Cell 15, 511-521 (2004).

17. Kikuchi, A. Roles of Axin in the Wnt signalling pathway. Cell Signal. 11, 777-788 (1999).

18. Yan, Y. et al. Axin2 controls bone remodeling through the beta-catenin-BMP signaling pathway in adult mice. J. Cell Sci. 122, 3566-3578 (2009).

19. $\mathrm{Yu}, \mathrm{H}$. M. et al. The role of Axin2 in calvarial morphogenesis and craniosynostosis. Development 132, 1995-2005 (2005).

20. Chia, I. V. \& Costantini, F. Mouse axin and axin2/conductin proteins are functionally equivalent in vivo. Mol. Cell Biol. 25, 4371-4376 (2005).

21. Xie, R., Jiang, R. \& Chen, D. Generation of Axin1 conditional mutant mice. Genesis 49, 98-102 (2011).

22. Song, L. et al. Loss of wnt/beta-catenin signaling causes cell fate shift of preosteoblasts from osteoblasts to adipocytes. J. Bone Miner. Res. 27, 2344-2358 (2012).

23. Zhang, $C$. et al. Inhibition of Wnt signaling by the osteoblast-specific transcription factor Osterix. Proc. Natl. Acad. Sci. USA 105, 6936-6941 (2008).

24. Krishnan, V., Bryant, H. U. \& Macdougald, O. A. Regulation of bone mass by Wnt signaling. J. Clin. Investig. 116, 1202-1209 (2006).

25. Glass, D. A. 2nd \& Karsenty, G. In vivo analysis of Wnt signaling in bone. Endocrinology 148, 2630-2634 (2007).

26. Chen, $M$. et al. Inhibition of beta-catenin signaling causes defects in postnatal cartilage development. J. Cell Sci. 121, 1455-1465 (2008).

27. Gori, F. et al. The expression of osteoprotegerin and RANK ligand and the support of osteoclast formation by stromal-osteoblast lineage cells is developmentally regulated. Endocrinology 141, 4768-4776 (2000).

28. Udagawa, N. et al. Osteoprotegerin produced by osteoblasts is an important regulator in osteoclast development and function. Endocrinology 141, 3478-3484 (2000).

29. Yasuda, H. et al. Osteoclast differentiation factor is a ligand for osteoprotegerin/ osteoclastogenesis-inhibitory factor and is identical to TRANCE/RANKL. Proc. Natl. Acad. Sci. USA 95, 3597-3602 (1998).

30. Wang, B. et al. Chondrocyte beta-catenin signaling regulates postnatal bone remodeling through modulation of osteoclast formation in a murine model. Arthritis Rheumatol. 66, 107-120 (2014).

31. Bronckers, A. L. et al. Phagocytosis of dying chondrocytes by osteoclasts in the mouse growth plate as demonstrated by annexin-V labelling. Cell Tissue Res. 301, 267-272 (2000).

32. Knowles, H. J. et al. Chondroclasts are mature osteoclasts which are capable of cartilage matrix resorption. Virchows Arch. 461, 205-210 (2012).

33. Tonna, S. et al. Chondrocytic ephrin B2 promotes cartilage destruction by osteoclasts in endochondral ossification. Development 143, 648-657 (2016).

34. Hayman, A. R. et al. Mice lacking tartrate-resistant acid phosphatase (Acp 5) have disrupted endochondral ossification and mild osteopetrosis. Development 122, 3151-3162 (1996).

35. Li, J. et al. RANK is the intrinsic hematopoietic cell surface receptor that controls osteoclastogenesis and regulation of bone mass and calcium metabolism. Proc. Natl. Acad. Sci. USA 97, 1566-1571 (2000).

36. Dougall, W. C. et al. RANK is essential for osteoclast and lymph node development. Genes Dev. 13, 2412-2424 (1999).

37. Oh, J. H. et al. Chondrocyte-specific ablation of Osterix leads to impaired endochondral ossification. Biochem. Biophys. Res. Commun. 418, 634-640 (2012).

38. Zhou, X. et al. Multiple functions of Osterix are required for bone growth and homeostasis in postnatal mice. Proc. Natl. Acad. Sci. USA 107, 12919-12924 (2010).

39. Zhu, M. et al. Activation of $\beta$-catenin signaling in articular chondrocytes leads to osteoarthritis-like phenotype in adult $\beta$-catenin conditional activation mice. J. Bone Miner. Res. 24, 12-21 (2009).

Open Access This article is licensed under a Creative Commons Attribution 4.0 International License, which permits use, sharing, adaptation, distribution and reproduction in any medium or format, as long as you give appropriate credit to the original author(s) and the source, provide a link to the Creative Commons license, and indicate if changes were made. The images or other third party material in this article are included in the article's Creative Commons license, unless indicated otherwise in a credit line to the material. If material is not included in the article's Creative Commons license and your intended use is not permitted by statutory regulation or exceeds the permitted use, you will need to obtain permission directly from the copyright holder. To view a copy of this license, visit http://creativecommons. org/licenses/by/4.0\%.

(c) The Author(s) 2020 